\title{
Dielectric properties, Impedance Analysis, and Electrical Conductivity of Ag Doped Radiation Grafted Polypropylene
}

\author{
L. A. Abdelwahab ${ }^{1}$, Amr El-Hag Ali', R. A. Zaghlool ${ }^{1}$, N. A. Mohsen ${ }^{2}$ \\ ${ }^{1}$ National Center for Radiation Research and Technology, Atomic Energy Authority, \\ P.O. Box 29, Nasr City, and ${ }^{2}$ Al-Azhar University, Nasr city, Cairo, Egypt
}

$\mathbf{T}$

HE possibility of obtaining relatively high dielectric constant polymer-metal composite by incorporating silver nanoparticles in a polypropylene (PP) polymer matrix using grafting and successive reduction method was demonstrated. Frequency and temperature dependence of AC conductivity and dielectric constant $\left(\varepsilon^{\prime}\right)$ and dielectric loss $(\varepsilon ")$ in pure polypropylene (PP), polypropylene grafted acrylic acid and styrene comonomer [PP-g-(AAc-co-Sty)], and PPg-(AAc-co-Sty)-Ag nanocomposite are studied in the frequency range $50 \mathrm{~Hz}-5 \mathrm{MHz}$ and in the temperature range $30-70^{\circ} \mathrm{C}$. The experimental results show that the polarity of PP is weak due to deficiency of polar groups. The values of $\varepsilon$ ' and $\varepsilon$ " increased dramatically with the addition of silver nanoparticles in polypropylene. The obtained AC conductivity follows the correlated barrier-hopping model ( $\mathrm{CBH}$ ) since the frequency exponent (s) and $\mathrm{AC}$ conductivity are both frequency and temperature dependent. The calculated value of the maximum barrier height $\mathrm{W}_{\mathrm{m}}$ for PP-g-(AAc-co-Sty)-Ag is $0.242 \mathrm{eV}$. The dielectric relaxation process is studied in respect of the electric modulus formalism.

Keywords: Grafting; Polypropylene; Nanocomposite; Silver nanoparticles; Impedance; Electric modulus.

\section{Introduction}

The rapid development of electronic products and power equipment, functional materials with excellent thermal and electrical properties are highly desirable. Polymeric materials are attractive due to their light weight, flexibility, ease of processing, and most importantly low cost. Interesting electrical applications need the electrical conductivity of a polymeric matrix to be improved by the addition of conductive particles. The charge carriers density will increase leading to formation of a conductive or semiconductive composite (Elimat, 2015). Particularly filled composites are commonly used in several applications. This is because introducing fillers, in particular nanoparticles, enhances the thermal and electrical properties of the polymer composites. The addition of nano-fillers conforms to the chain length of the polymer and then reduces MaxwellWagner-Sillar type interfacial polarization originated from the differences in dielectric permittivity of the polymer and filler (Peter Barber, 2009). There is an interest in dielectric materials because of their wide range of applications in electronic and electrical industry.
High dielectric constant $\left(\varepsilon^{\prime}\right)$, dielectrics, are suitable materials for cable insulation, chargestorage capacitors, electrocaloric cooling, and artificial muscles (Xingyi Huang, 2016).

Polymer-based composites have been extensively studied as the nominee materials for the capacitive components sector due to their high dielectric constant and good mechanical flexibility (Yu DANG , 2011).

Polypropylene (PP) is a good choice as host matrix. It exhibits excellent thermomechanical and electrical properties. Moreover it is low-cost material (Yu DANG, 2011). Ag nanoparticles are usually used as nanofillers for polymer matrices because of their catalytic and antimicrobial activity, good conductivity, chemical stability, and the possibility to improve physical properties of the applied host polymer (Konstantinos Chrissafis 2013).

Functionalization of the PP sheets is achieved via direct radiation grafting of Acrylic acid and Styrene monomers onto the surface of PP sheets. Successive reduction method is the preferred method to incorporate Ag nanoparticles into the functionalized polymeric matrix. 
Characterization of dielectric and electrical properties of the prepared nanocomposite polymer is needed to confirm their suitability as flexible-type polymeric nanodielectrics for the microelectronic device applications (Ram Jeewan Sengwa 2017).

The present study is developed to investigate the temperature and frequency dependence of dielectric properties and $\mathrm{AC}$ conductivity of pristine PP, PP-g-(AAc-co-Sty), and PP-g-(AAcco-Sty)-Ag nanocomposite.

\section{Experimental}

Preparation of Samples:

Graft PP copolymer was obtained by grafting Acrylic acid and styrene monomers onto polypropylene backbone (commercially available sheets) by gamma radiation. Polypropylene graft copolymer, modified with silver nanoparticles (Ag), was obtained through successive reduction of silver nitrate $\left(\mathrm{AgNO}_{3}\right)$ (Amr El-Hag, Ali 2017). Typically, the reaction solutions were prepared by dissolving $1 \mathrm{~g}$ of $\mathrm{AgNO}_{3}$ in $50 \mathrm{~g}$ of distilled water. A piece of PP graft copolymer of size $(2 \times 2) \mathrm{cm}$ was immersed in the solution for 30 minutes. Then, reduction takes place by $\mathrm{NaBH}_{4}$. Successive loading of the metal nanoparticles is accomplished by repeating the mentioned method.

\section{Characterization}

Dielectric properties measurement is conducted using Hioki 3532-50 Hi Tester LCR analyzer by the disc shaped two probe method in the frequency range of $50 \mathrm{~Hz}-5 \mathrm{MHz}$ and temperature range from 30 to $70{ }^{\circ} \mathrm{C}$.

\section{$\underline{\text { Results and Discussion }}$}

The complex dielectric constant of a material is represented by a real $\left(\varepsilon^{\prime}\right)$ and imaginary $\left(\varepsilon^{\prime}\right.$, , dielectric loss) parts according to:

$\varepsilon=\varepsilon^{\prime}+\mathrm{i} \varepsilon^{\prime \prime}$

The experimental dielectric constant $\varepsilon^{\prime}$ is calculated using the following equations (Alok Agrawal, 2015):

$\varepsilon^{\prime}=\mathrm{C} \mathrm{d} / \varepsilon_{\mathrm{o}} \mathrm{A}$

$\varepsilon^{\prime \prime}=\varepsilon^{\prime} \tan \delta$

Where $d$ is the thickness of the investigated sample, $\varepsilon_{0}$ is the permittivity of free space $(8.854$ $\left.\times 10^{-12} \mathrm{~F} / \mathrm{m}\right), \mathrm{A}$ is the area of the disc electrode, $\mathrm{C}$ is the parallel capacitance, $\delta=90^{\circ}-\theta$ and $\theta$ is the phase angle.
Figures $(1,2)$ show the dependence of $\varepsilon$ ' and $\varepsilon "$ on frequency in the range from $50 \mathrm{~Hz}$ to $5 \mathrm{MHz}$ for pristine PP, PP-g-(AAc-co-Sty), and PP-g-(AAc-co-Sty)-Ag nanocomposite at room temperature. Polymer could be polarized as a response of applying electric field, at lower temperatures, relaxations occur due to the orientation of smaller polar groups such as side groups or local segments of chains in polymer. The polarity of PP is weak due to deficiency of polar groups (Yu DANG 2011), and the dielectric response is also very weak and frequency independent as shown in Fig. 1. The dielectric constant and dielectric loss are increased in PP-g-(AAc-co-Sty) sample owing to the polar carboxylic groups of the graft copolymer. The addition of conductive Ag nanoparticles leads to the increase in dielectric constant (Figs. 1\& 2 ). This is due to the formation of a conducting percolation network of the conductive fillers in the polymer matrix. Also, the dielectric relaxation of the PP-g-(AAc-co-Sty) matrix and the interface of the composite is slow. The AC-conduction loss that dominates over other types of losses present in the polymer e.g. dipole segmental losses and orientation of the polar group lead to the high values of $\varepsilon$ ' and $\varepsilon$ " (Safenaz M. Reda 2012). Inspection of results reveals that PP-g(AAc-co-Sty)-Ag nanocomposite indicates their better ability to store electric energy under the influence of alternative electric field. The values of dielectric constant $(\varepsilon ', \varepsilon$ ') for the samples at certain frequencies are demonstrated in Table (1) for comparison.

The dielectric properties of composites at a low frequency can be explained by interfacial polarization or Maxwell- Wagner-Sillars effect. The polarization spends much more time than other dielectric processes because of the inertia of formed dipoles. Hence, the interfacial polarization occurs at a low frequency, and $\varepsilon$ ', $\varepsilon$ ', decrease rapidly when frequency increases (Figs. 1,2). At higher frequencies, the response from PP matrix becomes dominant, the dielectric constant is comparatively independent of frequency and is generally lower than that at a low frequency ( $\mathrm{Yu}$ DANG, 2011).

Figures 3, 4 show that $\underline{\varepsilon^{\prime} \text { and } \varepsilon^{\prime \prime}}$ increase greatly with increasing temperature up to frequency $10 \mathrm{kHz}$, after that its values increase slightly with increasing the frequency. The effect of frequency on dielectric constant $\left(\underline{\left.\varepsilon^{\prime}, \varepsilon^{\prime \prime}\right)}\right.$ with increasing temperature is shown in Figs. $(5,6)$. 


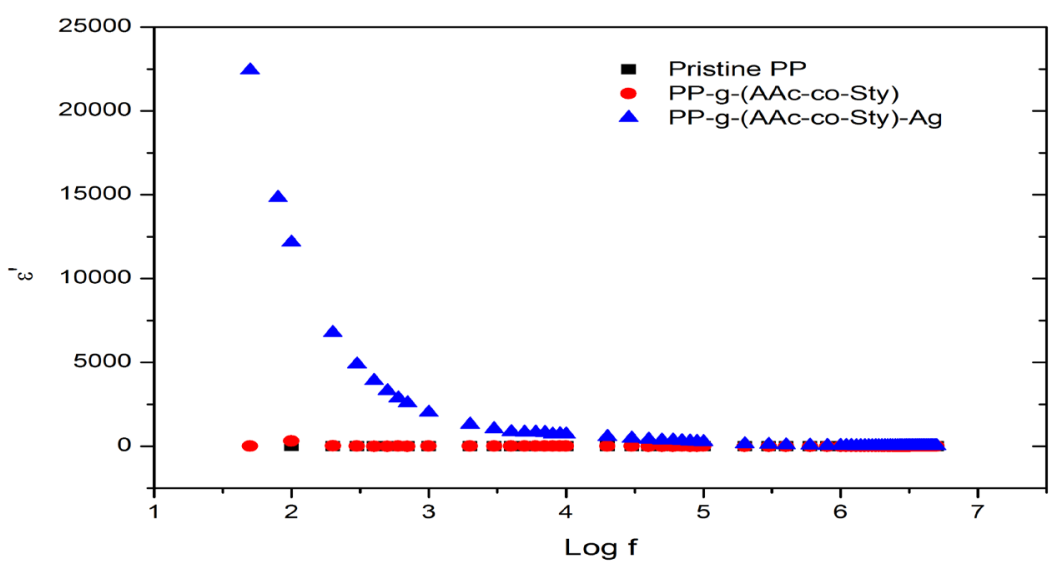

Fig.1. The effect of grating and metal doping on the dielectric constant of polypropylene.

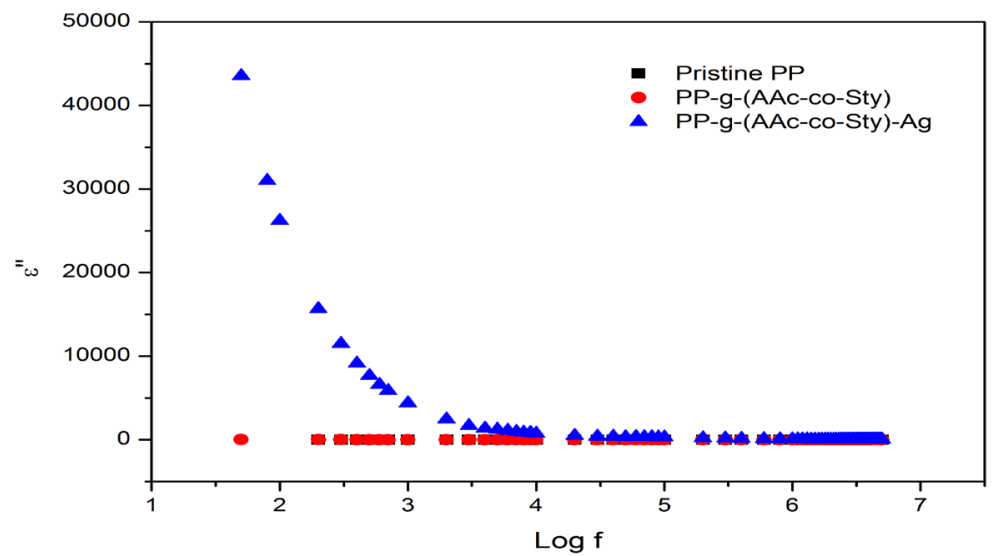

Fig .2. The effect of grating and metal doping on the dielectric loss of polypropylene.

TABLE 1 . The real and imaginary parts of dielectric constants of Pristine PP, PP-g-(AAc-co-Sty), and PP-g-(AAcco-Sty) - Ag samples at room temperature and certain frequencies.

\begin{tabular}{|c|c|c|c|c|c|c|}
\hline \multirow[b]{2}{*}{ Sample } & \multicolumn{2}{|c|}{$1 \mathrm{kHz}$} & \multicolumn{2}{|c|}{$10 \mathrm{kHz}$} & \multicolumn{2}{|c|}{$100 \mathrm{kHz}$} \\
\hline & $\varepsilon '$ & $\varepsilon "$ & $\varepsilon '$ & $\varepsilon "$ & $\varepsilon '$ & $\varepsilon "$ \\
\hline Pristine PP & 2.311 & 0.302 & 0.867 & 0.179 & 1.895 & 0.564 \\
\hline PP-g-(AAc-co-Sty) & 6.386 & 0.509 & 9.008 & 2.379 & 6.250 & 1.853 \\
\hline PP-g-(AAc-co-Sty)-Ag & 2007.017 & 4363.073 & 719.057 & 766.666 & 256.381 & 299.904 \\
\hline
\end{tabular}

Egypt. J. Rad. Sci. Applic., Vol. 30, No.1(2017) 


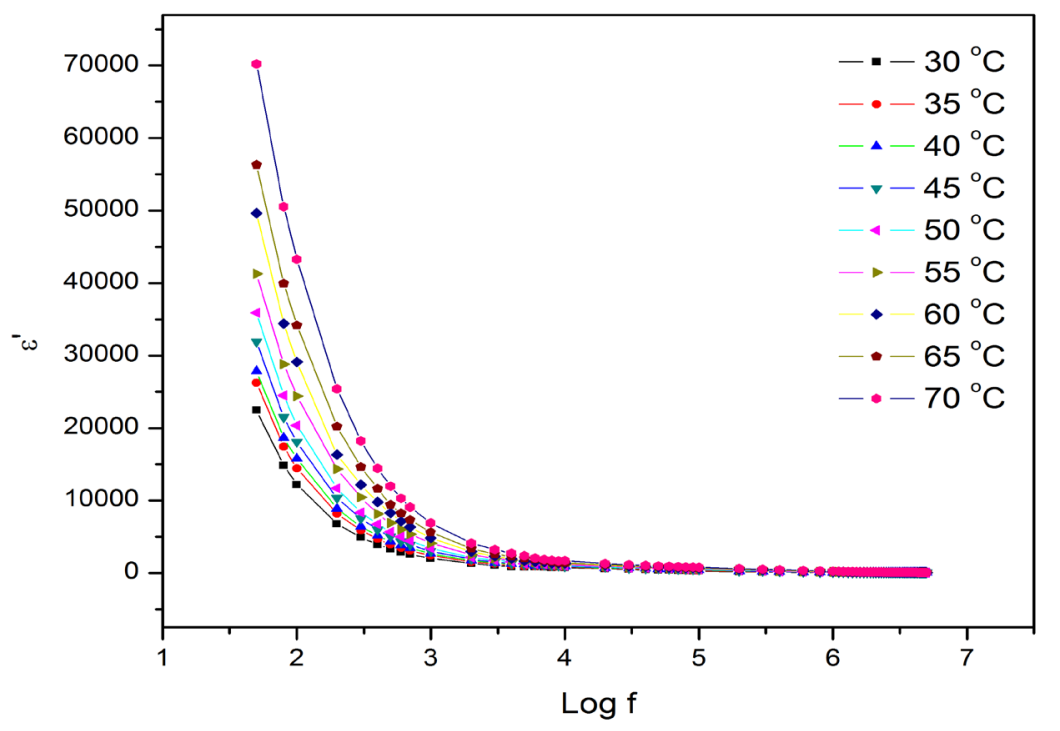

Fig .3. Effect of temperature on dielectric permittivity (É) of PP- g- (AAC-co-sty)- Ag nanocomposite with increasing frequency .

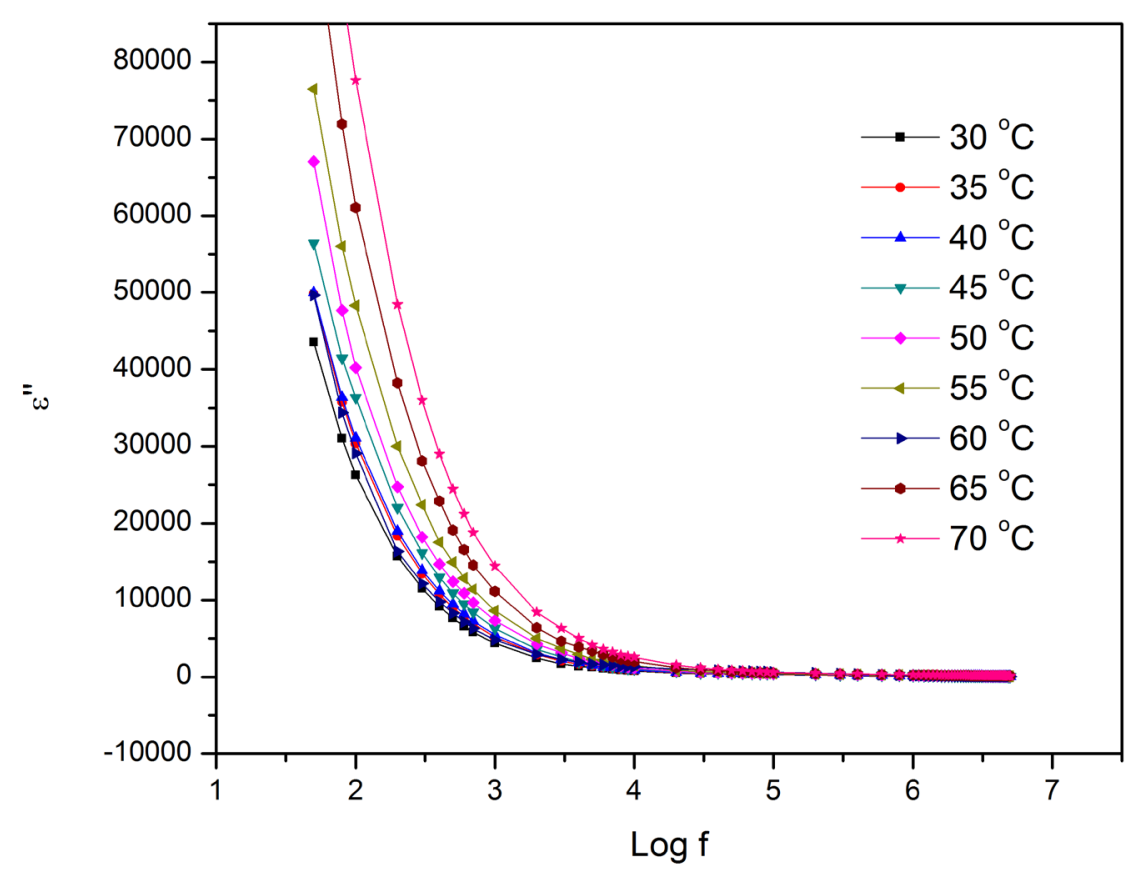

Fig .4. Effect of temperature on dielectric permittivity (๕̌) of PP- g- (AAC-co-sty)- Ag nanocomposite with increasing frequency. 


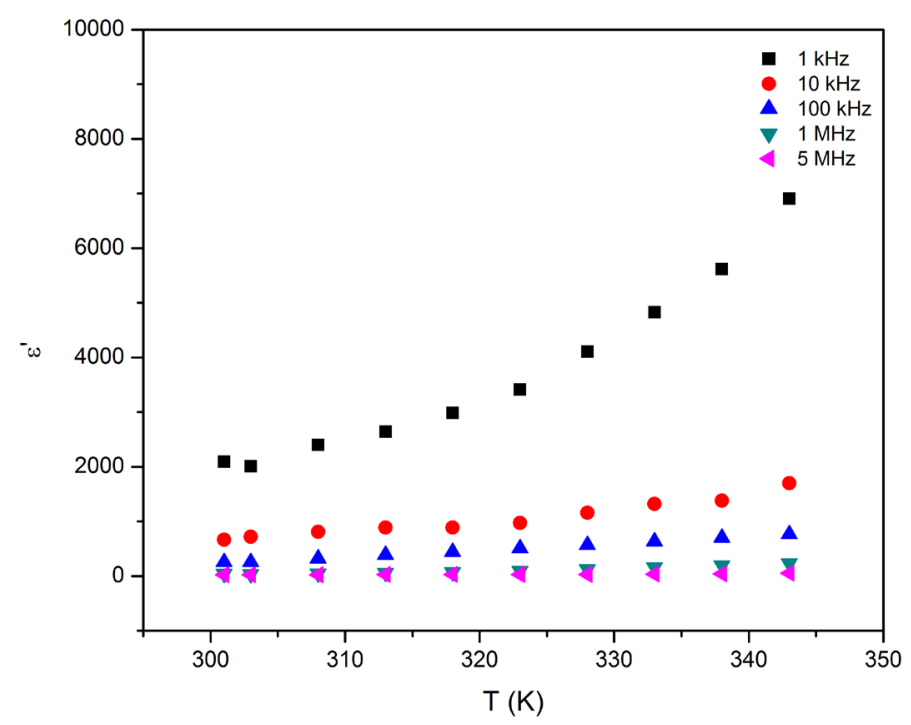

Fig .5. Effect of frequency on dielectric permittivity (实 of PP- g- (AAC-co-sty)- Ag nanocomposite with increasing temperateure.

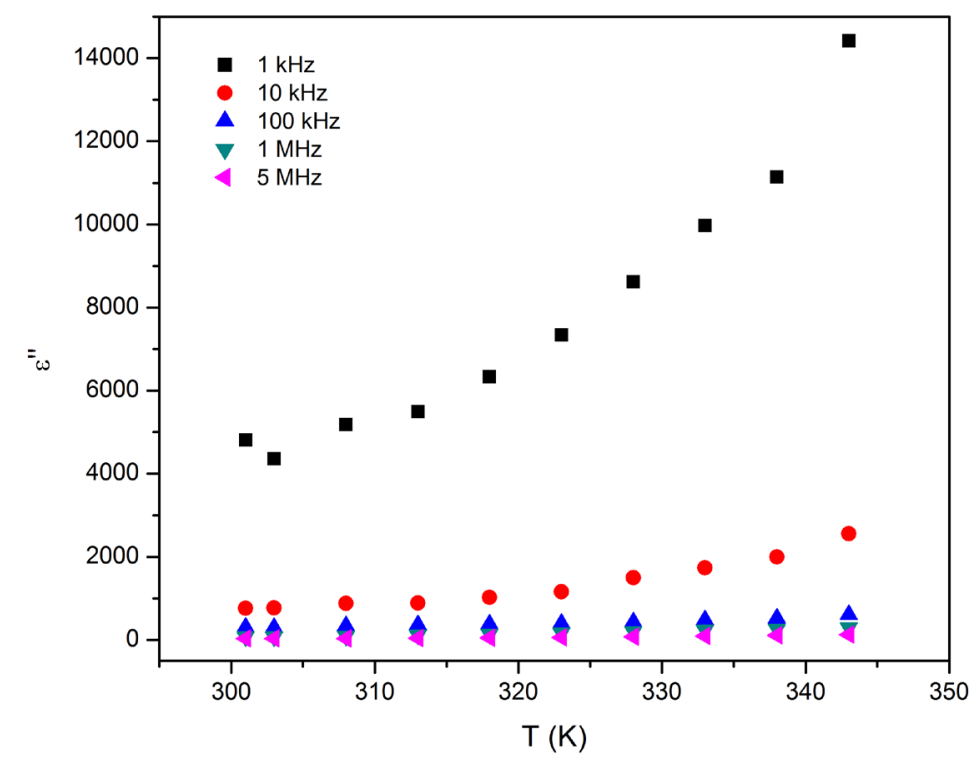

Fig .6. Effect of frequency on dielectric $\operatorname{loss}(\tilde{\varepsilon})$ of PP- g- (AAC-co-sty)- Ag nanocomposite with increasing temperateure . 
The imaginary part of dielectric constant $(\varepsilon ')$ is expressed according to Zulfequar (2015) as follows:

$$
\varepsilon^{\prime \prime}=\mathrm{B} \omega^{\mathrm{m}}
$$

Where $\mathrm{B}$ is a constant and $\mathrm{m}$ is the frequency power factor.

Plotting $\ln \varepsilon$ " vs. $\ln \omega$ as shown in Fig. (7), one can calculate the value of the power factor $m$ from the slope of these lines. The variation of $m$ with temperature is shown in Fig. (8).

The barrier height $\left(\mathrm{W}_{\mathrm{m}}\right)$, the energy required to move the electron from a site to infinity, is calculated according to the relation formulated by Stenels (1975):

$\mathrm{m}=\frac{-4 T}{W_{m}}$

Where $\mathrm{k}$ is Boltzmann constant and $\mathrm{T}$ is the temperature in Kelvin.

The obtained value of the barrier height is found to be $0.242 \mathrm{eV}$.

\section{Complex Impedance Analysis:}

The variation of real (Z') and imaginary (Z') parts of the dielectric function with frequency and temperature of PP-g-(AAc-co-Sty) - Ag nanocomposite is shown in Fig. 9\&10. The high values of $Z^{\prime}$ at low frequencies are due to space charge polarization. It is evident that the impedance curves merge at higher frequencies, denoting that the real part of impedance (Z') is slightly dependent on temperature at high frequencies. This behavior indicates the dominance of space charge polarization, which occurs at low frequencies and also suggests a reduction in the barrier. The decrease in impedance at higher frequencies may be due to the hopping of electrons between localized ions (N. Padmamalini 2016).

The effect of temperature on the impedance of PP-g-(AAc-co-Sty)-Ag sheet can be clearly understood from the Cole-Cole plot ( $Z$ ' versus Z') shown in Fig. 11. These plots exhibit two distinguished semicircles: the characteristic depressed semicircle at high frequencies and the quasi-linear response at low frequencies. According to previously reported studies, a depressed semicircle describes properties of the bulk material, and the quasi-linear response at

Egypt. J. Rad. Sci. Applic., Vol. 30, No.1(2017) low frequencies is associated with the interfacial polarization and/or the surface and electrode effects (J. Betzabe Gonz'alez-Campos, 2012, Alexandra Neagu, 2015). The intercepts of the semicircles on low frequency end gives the bulk resistance $\left(\mathrm{R}_{\mathrm{b}}\right)$ of the sample at different temperatures. The frequency at the maximum of the semicircle is equal to $\omega=1 / \mathrm{RC}$. The relaxation time is given by $\tau=\mathrm{RC}$. Therefore $\omega$ $\tau=1$ (N. Padmamalini, 2016). The shift of the characteristic semicircle to higher frequencies with increasing temperature indicates enhanced dc conductivity with increasing temperature.

\section{Complex Electric Modulus}

Electric modulus formalism is the powerful tool to study conductivity relaxation process. The advantage of using the electric modulus to interpret bulk relaxation properties is that changes in the large values of permittivity and conductivity at low frequencies are minimized. This way, the familiar difficulties of electrode nature and contact, space charge injection phenomena and absorbed impurity conduction effects, which appear to obscure relaxation in the permittivity presentation, can be solved or even ignored (Alexandra Neagu ,2015).

The complex electric modulus $\mathrm{M}^{*}$ can be represented according to a formula by $\mathrm{S}$. Saha( 2015):

$$
\begin{aligned}
& \left.\mathrm{M}^{*}=\mathrm{M}\right\rangle+\mathrm{i} \text { 〉〉 } \\
& M>=\varepsilon^{\prime} /\left(\varepsilon^{\prime 2}+\varepsilon^{\prime \prime}\right) \quad \& M^{\prime \prime}=\varepsilon^{\prime \prime} /\left(\varepsilon^{\prime 2}+\varepsilon^{\prime \prime 2}\right)
\end{aligned}
$$

$\mathrm{M}>$ and $\mathrm{M} \gg$ are the real and imaginary parts of the dielectric modulus.

Real $(\mathrm{M}>)$ and imaginary $(\mathrm{M} \gg)$ modulus spectra at different temperatures for PP-g-(AAcco-Sty)-Ag sample have been shown in Figs. $12 \& 13$, respectively. Comparisons of $\varepsilon$ ' (f) and M'(f) spectra have been used to distinguish localized dielectric relaxation processes from longrange conductivity (Alexandra Neagu, 2015). A conductivity relaxation is indicated by the presence of a peak in the spectra of M'(f) and no peak in the corresponding plot of $\varepsilon$ "'(f), while the dielectric relaxation gives maxima both in the imaginary part of permittivity $\varepsilon$ '(f) and of the dielectric modulus M''(f) spectra. The plotin Fig. 13 exhibits pronounced relaxation peaks for M', and no peaks in the corresponding plots of imaginary part of dielectric constant $\varepsilon$ '(f) in Fig. 4 which manifests long-range conductivity. 


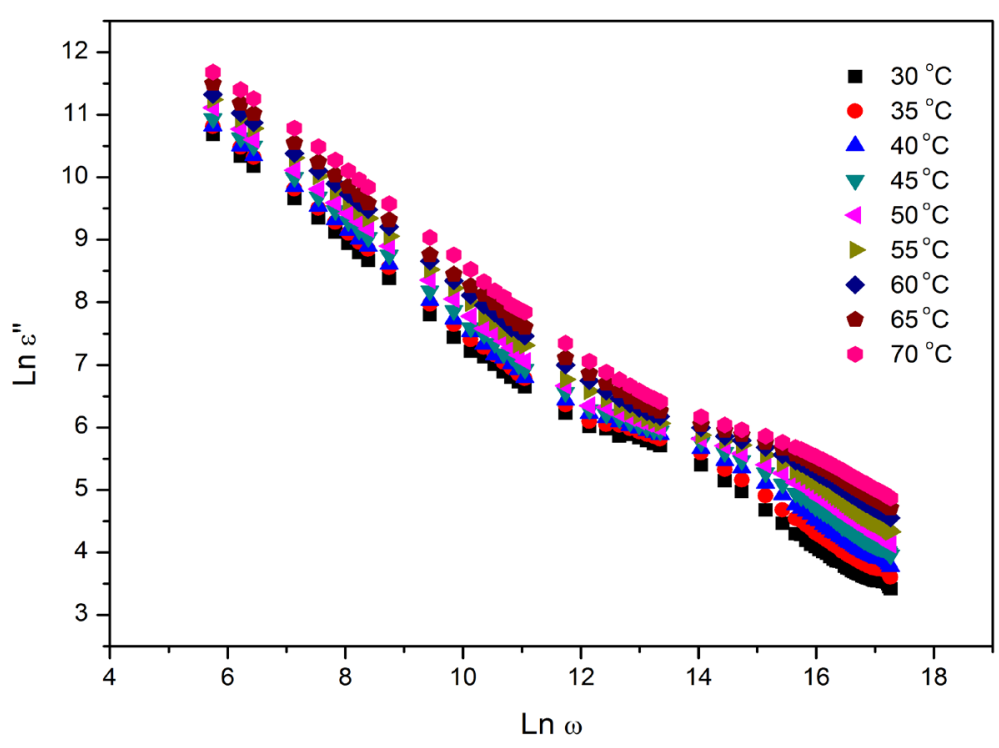

Fig .7. Frequency dependence of In $\grave{\varepsilon}$ of PP- g- (AAC-co-Sty)- Ag nanocomposite at temperateure .

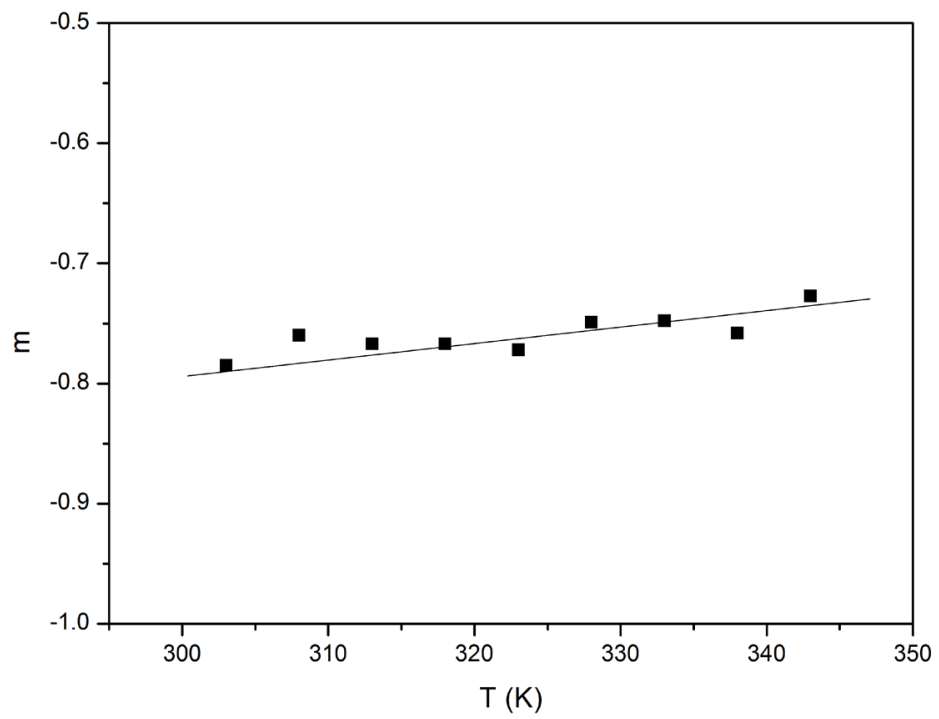

Fig .8. The temperatures dependence of the parameter m for PP- g- (AAC-co-Sty)- Ag nanocomposite . 


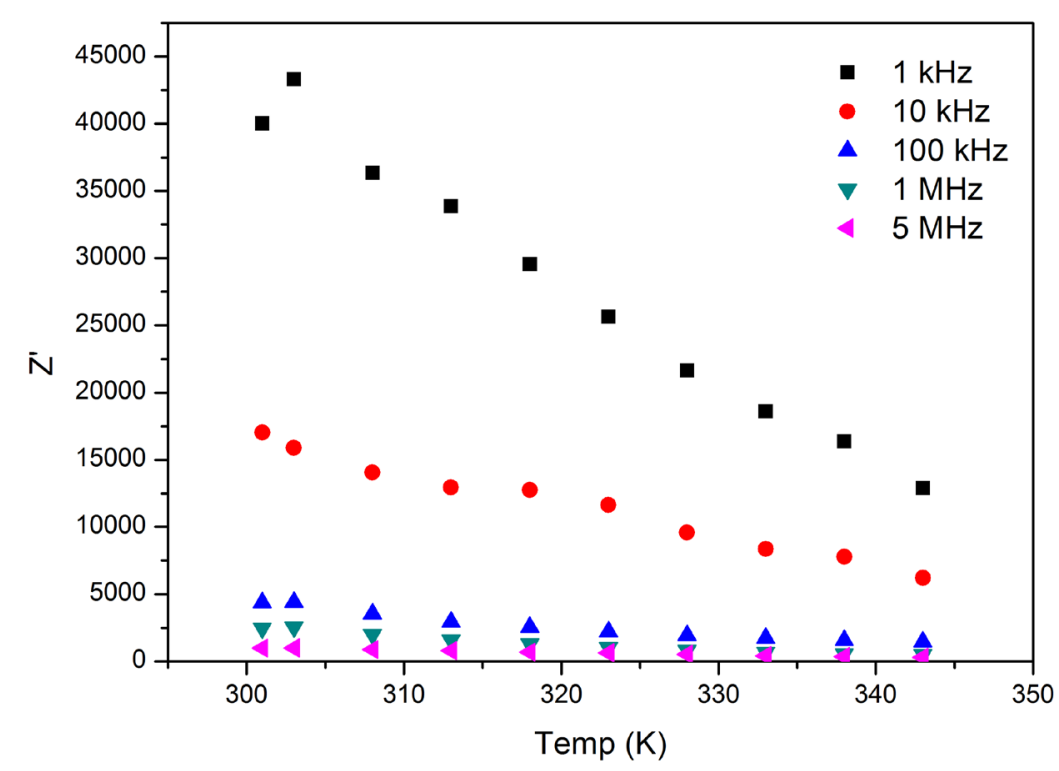

Fig .9. Effect of frequency on real part of complex impedance $Z$ of PP- g- (AAC-co-sty)- Ag nanocomposite measured asa function of temperature .

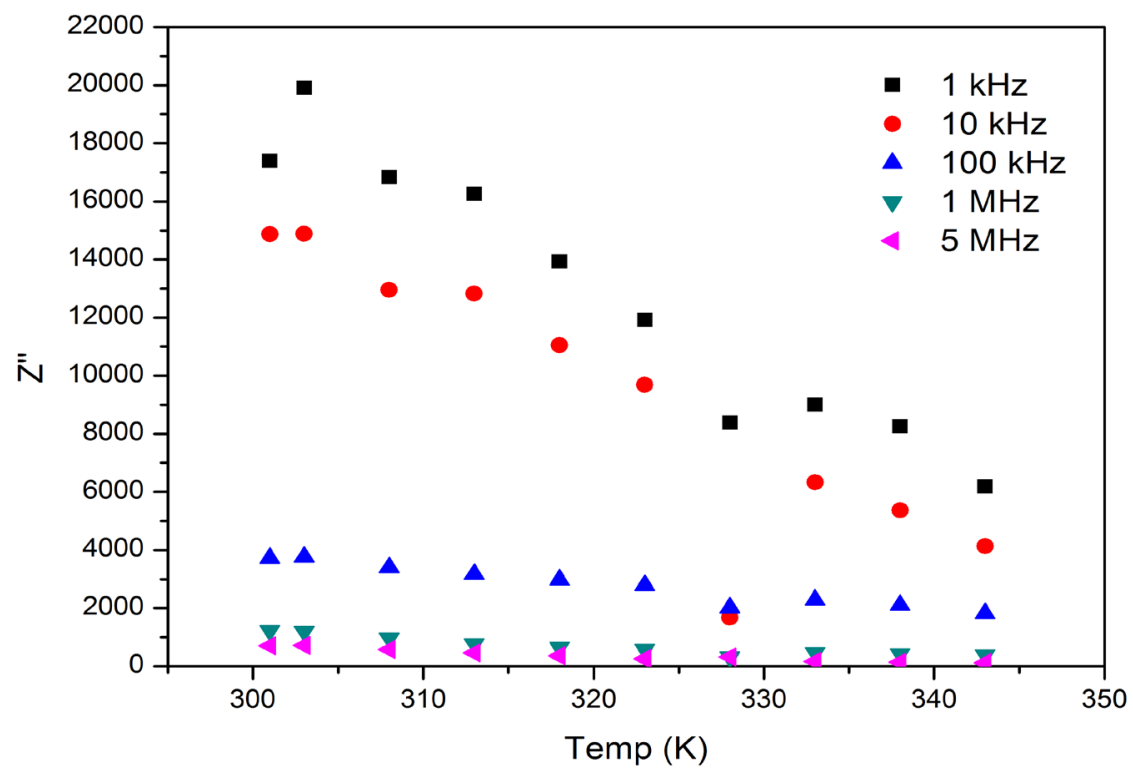

Fig .10. Effect of frequency on imaginary part of complex impedance $Z$ of PP- g- (AAC-co-sty)- Ag nanocomposite measured asa function of temperature . 


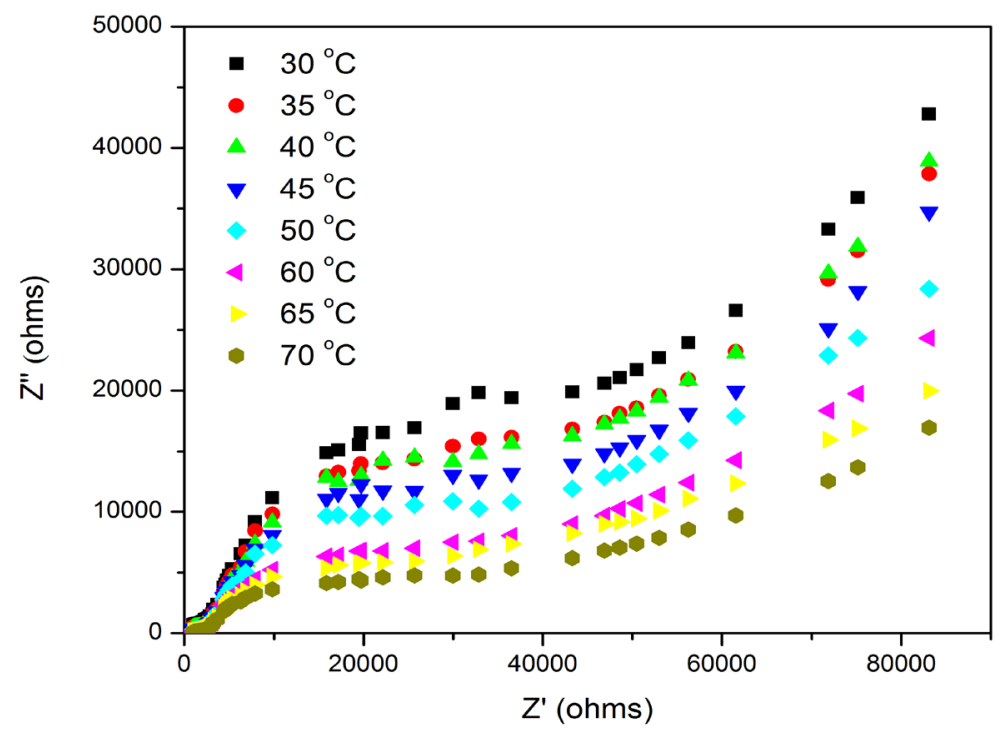

Fig .11. Cole - Cole plots of PP- g- (AAC-co-sty)- Ag nanocomposite : effect of Temperature .

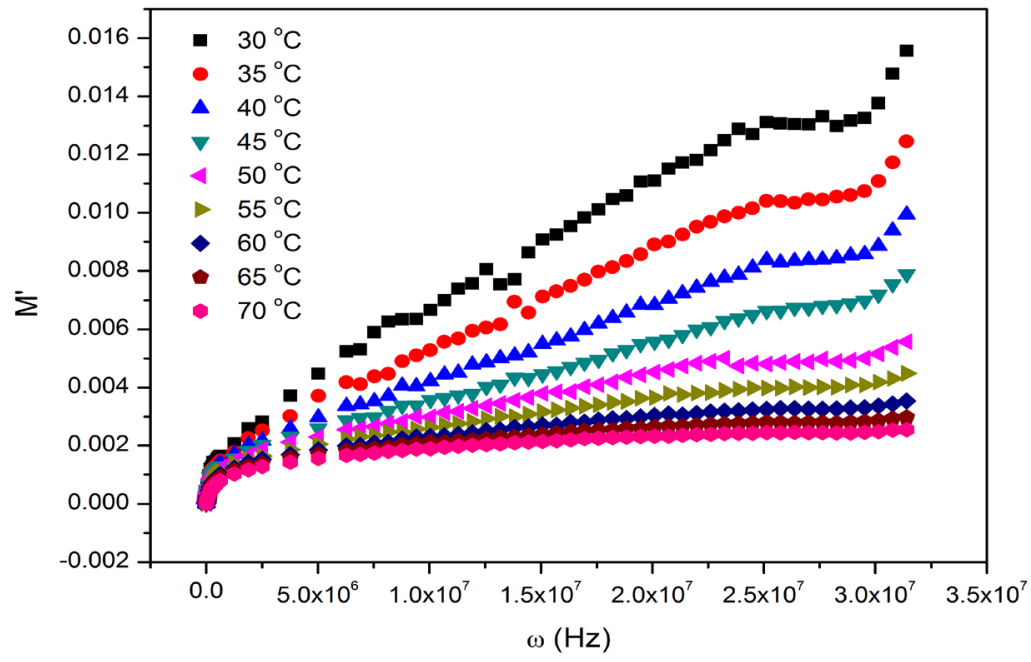

Fig.12. Effect of temperature on real part of Complex Modulus (M“) of PP- g- (AAC-co-sty)- Ag nanocomposites measured as a function of Frequency . 


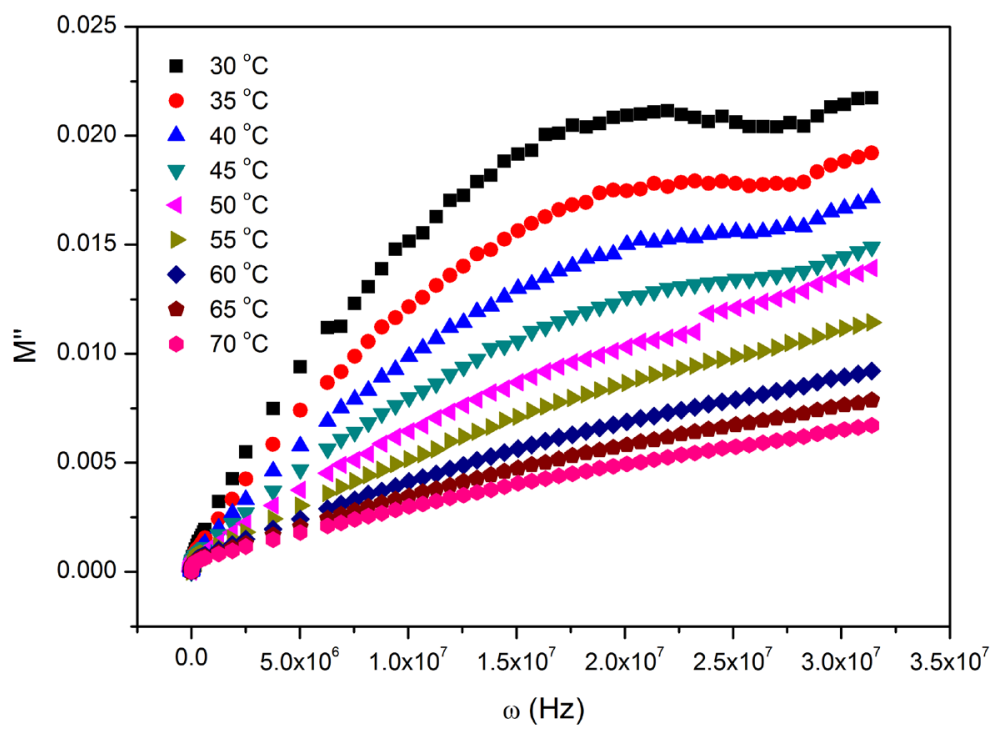

Fig .13. Effect of temperature on real part of Complex Modulus (M“) of PP- g- (AAC-co-sty)- Ag nanocomposites measured as a function of Frequency .

The low frequency side of the peak represents the frequencies for which charge carriers are mobile over a long range and can successfully hop from one defect site to another. The high frequency side of the peak signifies the frequencies for which the charge carries are confined to their potential wells and no long range motions are possible. Here the peaks shift towards higher frequencies as the temperature increases. As a consequence, it can be inferred that the relaxation time given by $\tau=1 / \omega_{\max }$ decreases as temperature increases. Therefore the relaxation process is temperature dependent. The plots also indicate a change in both $\mathrm{M}^{\prime \prime}{ }_{\max }$ as well as in $\omega_{\max }$, which denotes a change in $\mathrm{C}$ values with temperature $(\mathrm{N}$. Padmamalini, 2016).

\section{Electrical AC Conductivity:}

The ac conductivity $\sigma_{\text {ac }}(\omega)$ is expressed as (Zulfequar 2015):

$\sigma_{\mathrm{ac}}(\omega)=\sigma_{\mathrm{t}}(\omega)-\sigma_{\mathrm{dc}}(\omega)=A \omega^{\mathrm{s}}$

Where $\omega$ is the angular frequency, $\sigma_{t}(\omega)$ is the measured total electrical conductivity, $\sigma_{\mathrm{dc}}(\omega)$ is the dc electrical conductivity, $\mathrm{s}$ is the frequency exponent and $\mathrm{A}$ is constant dependent on temperature.

The ac conductivity is known to be a thermally activated transport process which could be described with the Arrhenius equation (Sergei Bronnikov, 2017):

Egypt. J. Rad. Sci. Applic., Vol. 30, No.1(2017)

$$
\sigma_{\mathrm{ac}}=\sigma_{0} \exp \left(-\mathrm{E}_{\mathrm{a}} / \mathrm{kT}\right)
$$

where $E_{a}$ is the activation energy of the mobile charge carriers, $\mathrm{k}$ is the Boltzmann constant, and $\mathrm{T}$ is the absolute temperature.

It is evident from Figs. 14-16 that the ACconductivity is both frequency and temperature dependent and enhanced with an increase of both the frequency and the temperature. This can be accounted for by assuming that there may be charge carriers, which can be transported by hopping through the defect sites along the polymer chain (Safenaz M. Reda, 2012). The temperature dependence of the frequency exponent (s) demonstrates that the correlated barrier-hopping $(\mathrm{CBH})$ model is the most probable conduction mechanism. In the $\mathrm{CBH}$ model a temperature dependent exponent, $\mathrm{s}(\mathrm{T})$, is predicted, with s decreasing as the temperature increases and $\mathrm{s}$ increases towards unity as the temperature tends to zero (M. S. Hossain, 2007). The activation energy $E_{a}$ has been calculated from the slope of $\ln \sigma_{\mathrm{ac}}$ vs. $1000 / \mathrm{T}$ plot. The relation between $\mathrm{E}_{\mathrm{a}}$ and frequency is illustrated in Fig. (17). The values of the ac activation energy in low frequencies is in a good agreement with those of the barrier height in the same range of frequency. 


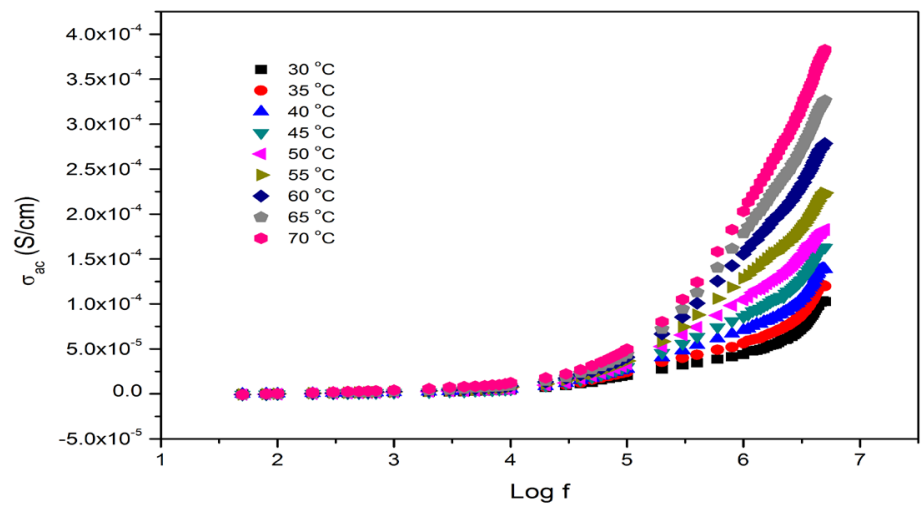

Fig.14. Effect of temperature on the AC conductivity of PP-g-(AAC-co-sty)-Ag nanocomposites with increasing frequency .

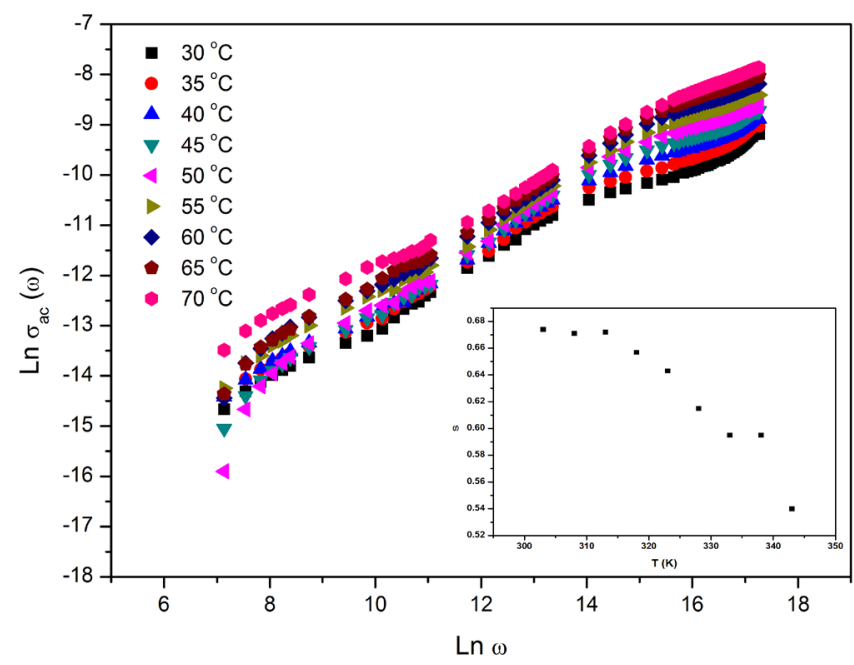

Fig.15. Frequency dependence of $\operatorname{In} \sigma_{\text {ac }}(\omega)$ at different temperatures. The inset figure shows the temperature dependence of the ofs for PP-g- (AAC-co-sty)- Ag nanocomposites.

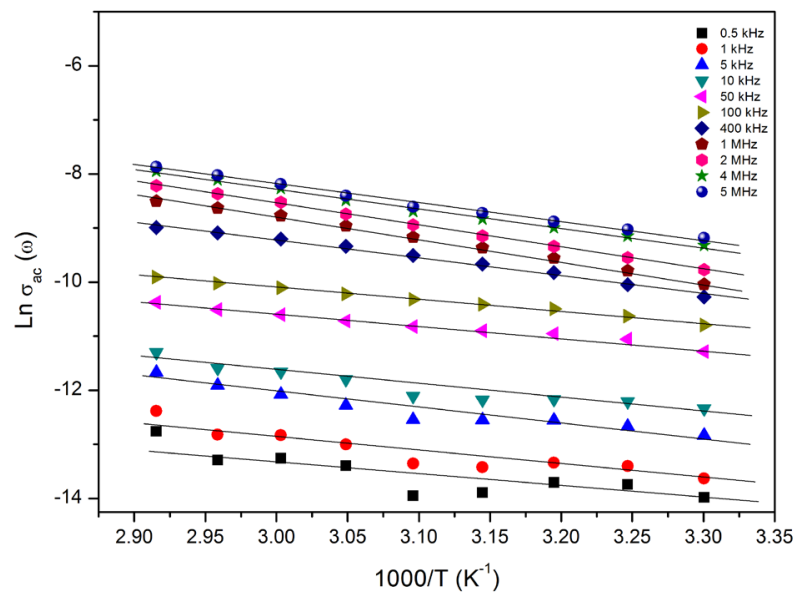

Fig.16. AC conductivity as a function of reciprocal temperature for PP- g- (AAC-co-sty)- Ag nanocomposite at different frequencies. 


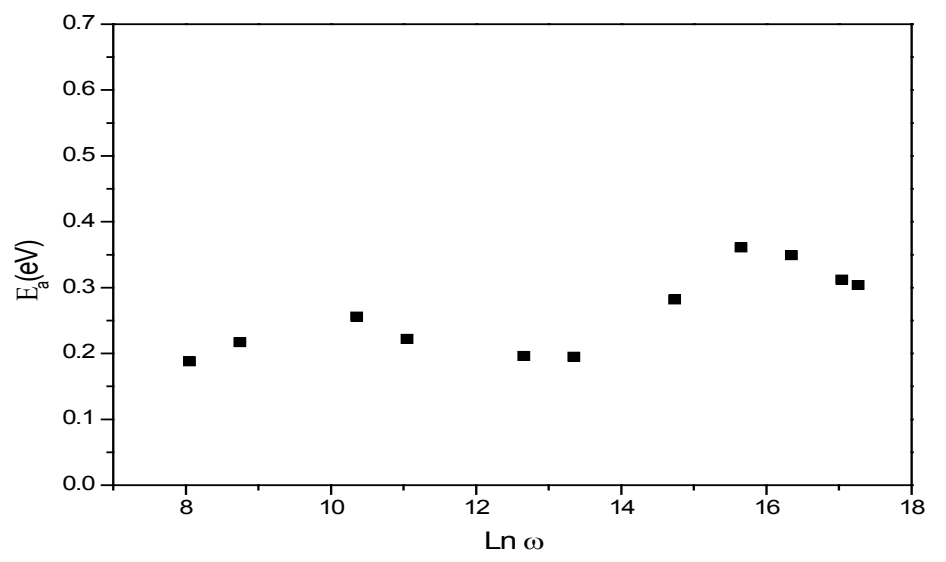

Fig.17. Frequency depence of activation energy $E_{a}(\omega)$ for PP- g- (AAC-co-sty)- Ag nanocomposite .

It can be seen that the conductivity of PP-g(AAc-co-Sty)-Ag nanocomposite increases as the frequency increases. The frequency dependence of the ac-conductivity is considered to be a result of interface charge polarization (or MaxwellWagner-Sillars effect) and intrinsic electric dipole polarization. This phenomenon appears in heterogeneous systems such as metal-polymer composites due to the accumulation of mobile charges at the interfaces and the formation of large dipoles on metal particles or clusters (Safenaz M. Reda 2012).

\section{Conclusion}

The dielectric study of pristine PP, PPg-(AAc-co-Sty), and PP-g-(AAc-co-Sty)-Ag nanocomposite, in the temperature range $30-70$ ${ }^{\circ} \mathrm{C}$ and frequency range $50 \mathrm{~Hz}-5 \mathrm{MHz}$ leads to the following conclusions. The dielectric response of PP is very weak and frequency independent. The addition of silver nanoparticles to polypropylene has increased the real and imaginary parts of dielectric constant dramatically. This behavior may be due to the ac-conduction loss that dominates over other types of losses present in the polymer e.g. dipole segmental losses and orientation of the polar group. The prepared composite show enhanced dc-conductivity with increasing temperature since Cole-Cole plots exhibit a shift of the characteristic semicircle to higher frequencies with increasing temperature. A conductivity relaxation is indicated by the presence of a peak in the spectra of $\mathrm{M}^{\text {' }}(\mathrm{f})$ and no peak in the corresponding plot of $\varepsilon$ ''(f). The correlated barrier-hopping (CBH) model is applicable to explain the observed behavior

Egypt. J. Rad. Sci. Applic., Vol. 30, No.1(2017) of the ac-conductivity since it is both frequency and temperature dependent and enhanced with an increase of both the frequency and the temperature.

\section{References}

Alexandra Neagu, L. C., Mirela Airimioaei, Ana Cazacu, Adrian Cernescu, Liliana Mitoseriu (2015) Impedance spectroscopy characterization of relaxation mechanisms in gold-chitosan nanocomposites. Composites: Part B, 71, 210.

Alok Agrawal, A. S. (2015) Thermal and dielectric behaviour of polypropylene composites reinforced with ceramic fillers. J Mater Sci: Mater Electron, 26, 103.

Amr El-Hag Ali, L. A. Wahab., R. A. Zaghlool, N. A. Mohsen (2017) Physical Characterization of Ag Doped Radiation Grafted Polypropylene. ninth International Conference for Basic Sciences, In Press.

Elimat, Z. (2015) AC-impedance and dielectric properties of hybrid polymer composites. Journal of Composite Materials, 49, 3.

J. Betzabe Gonz'alez-Campos, E. P., Isaac C. Sanchez, J. Gabriel Luna-B'arcenas, Alejandro ManzanoRam'irez, Jes ' us Gonz'alez-Hern'andez, Yliana L'opez-Castro, and Rosa E. del R'10 (2012) Molecular Dynamics Analysis of PVA-AgnP Composites by Dielectric Spectroscopy. Journal of Nanomaterials, 1.

Konstantinos Chrissafis, E. P., Dimitra Gkogkou, Dimitrios Bikiaris (2013) Effect of Cu-nanofibers and Ag-nanoparticles on syndiotactic polystyrene thermal stability and on its decomposition mechanism. Thermochimica Acta, 561, 26. 
M. S. Hossain, R. I., K. A. Khan (2007) Electrical conduction mechanism and dielectric properties of vanadium doped $\mathrm{ZnTe}$ thin films. Journal of Optoelectronics and Advanced Materials, 9, 2192.

N. Padmamalini, K. A. (2016) Impedance and modulus spectroscopy of $\mathrm{ZrO}_{2}-\mathrm{TiO}_{2}-\mathrm{V}_{2} \mathrm{O}_{5}$ Nanocomposite. Karbala International Journal of Modern Science, 2, 271 .

Peter Barber, S. B., Yogesh Anguchamy, Shushan Gong, Arief Wibowo, Hongsheng Gao, Harry J. Ploehn and Hans-Conrad zur Loye (2009) Polymer Composite and Nanocomposite Dielectric Materials for Pulse Power Energy Storage. Materials, 2, 1697.

Ram Jeewan Sengwa, S. C. (2017) Dielectric and electrical properties of $\mathrm{PEO}-\mathrm{A} 12 \mathrm{O} 3$ Nanocomposites._Journal of Alloys and Compounds. doi: 10.1016/j.jallcom.2017.01.155.

S. Saha, A. N., A.K.Meikap, S.K.Pradhan (2015) Electric modulus formalism and electrical transport property of ball mill synthesized nanocrystalline $\mathrm{Mn}$ doped $\mathrm{ZrO} 2$ solid solution. Physica B, 479, 67.
Safenaz M. Reda, S. M. A.-G. (2012) Synthesis and Electrical Properties of Polyaniline Composite with Silver Nanoparticles. Advances in Materials Physics and Chemistry, 2, 75.

Sergei Bronnikov, A. P., Sergei Kostromin, Mihai Asandulesa, Vasile Cozan (2017) Electrical conductivity of polyazomethine/fullerene C60nanocomposites. Physics Letters A, 381, 796.

Stenels, J. M. (1975) The electrical properties of glass.

Xingyi Huang, C. Z. (2016) Polymer Nanocomposites: Electrical and Thermal Properties. Switzerland, Springer.

Yu DANG, Y. W., Yuan DENG, Mao LI, Ye ZHANG, Zhi-wei ZHANG (2011) Enhanced dielectric properties of polypropylene based composite using $\mathrm{Bi}_{2} \mathrm{~S}_{3}$ nanorod filler. Progress in natural science: Materials international, 21, 216.

Zulfequar, M. G. a. M. (2015) AC Conductivity Measurement of $\mathrm{Cd}_{5} \mathrm{Se}_{95-\mathrm{x}} \mathrm{Zn}_{\mathrm{x}}$ Chalcogenide Semiconductor Using Correlated Barrier Hopping Model. Acta Physica Polonica A, 128, 59.

(Received 27/6/2017; accepted 27/9/2017)

\section{" خصائص العزل الكهربائي وتحليل المعاوقة و الموصلية الكهربائية لمادة بولي برويلين المطعة إشعاعيا بالفضة العزي}

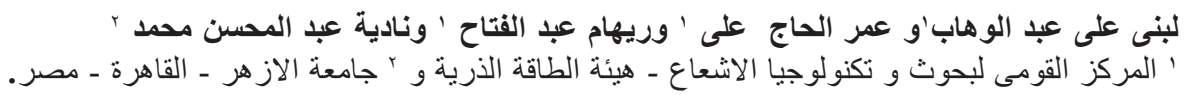

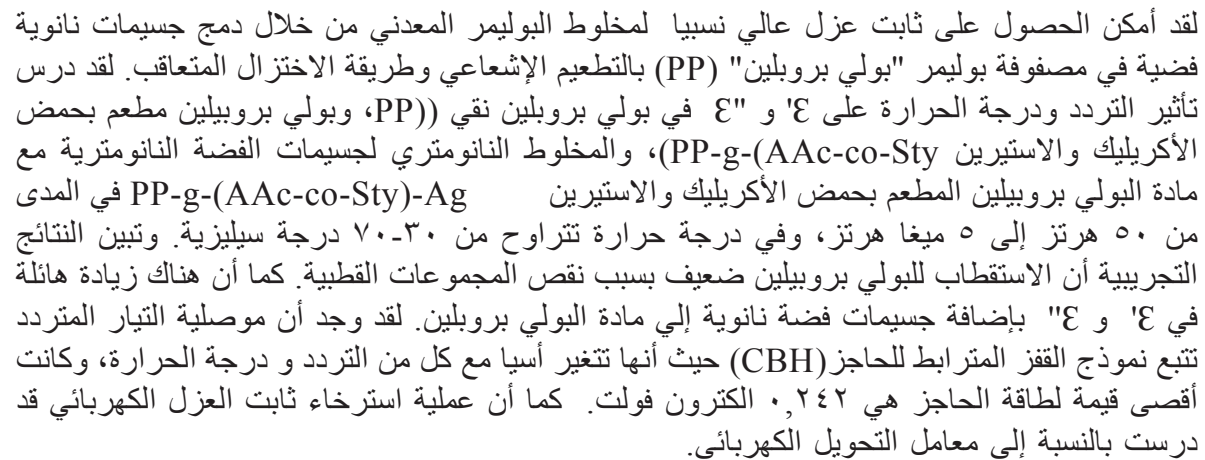

